\title{
SUPPLEMENTARY INFORMATION 1 \\ EPITOPE-BASED POTENTIAL VACCINE CANDIDATE FOR HUMORAL AND CELL MEDIATED IMMUNITY TO COMBAT SEVERE ACUTE RESPIRATORY SYNDROME CORONAVIRUS 2 PANDEMIC
}

\author{
Bratin Kumar Das, Debashree Chakraborty*
}

Biophysical and Computational Laboratory, Department of chemistry, National Institute of Technology Karnataka, Surathkal, Mangalore, 575025

*Corresponding Author Email: debashree@nitk.edu.in

\section{Methodology}

\section{Multiple Sequence Alignment and domain analysis}

The evolutionary diversity of various classes of corona-virus S-protein was primarily determined by Multiple sequence analysis (MSA). We used MEGA7 ${ }^{1,2}$ software for MSA and the Phylogenic tree was constructed by ClustalW tool ${ }^{3}$, employing neighbour joining algorithm with default parameters and 1,000 bootstrap replicas. In this paper, S-protein amino acid sequences of all coronavirus species ( $\alpha$-coronavirus, $\gamma$-coronavirus, $\beta$-coronavirus such as SARS-CoVid, MERS-CoVid and SARS$\mathrm{CoVid} 2, \delta$-coronavirus) were considered which cause severe lungs diseases in human or animal. Additionally, S-protein sequences of SARS-CoVid2 retrieved from most affected countries in the globe were included in MSA and Phylogenic tree analysis. All the sequences that are included in this study were retrieved from NCBI database (https://www.ncbi.nlm.nih.gov/).

Further, the major domains of spike glycoprotein of SARS-CoVid-2 were identified by Pfam database (https://pfam.xfam.org/).

\section{B-cell epitope prediction}

In order to design anti-SARS-CoVid2-nutralizing antibodies, the antigenic sites of S-protein were determined using IEDB (Immune-Epitope-Database and Analysis-Resource) tool (http://tools.iedb.org/main/). The linear B-cell epitopes were predicted by set-of physicochemical parameters such as the exposed surface propensity by Emini et. $\mathrm{al}^{4}$, hydrophilicity by Parker et. $\mathrm{al}^{5}$, flexibility by Karplus et. $\mathrm{al}^{6}$, antigenic propensity by Kolaskar et. $\mathrm{al}^{7}$ and $\beta$-turn propensity by Chou et. $\mathrm{a} \mathrm{l}^{8}$ etc. In addition, the linear epitopes were also predicted by Bepipred Linear epitope prediction method $^{9}$. The surface accessible probability $\left(\mathrm{S}_{\mathrm{n}}\right)$ of an amino-acid located at the $\mathrm{n}^{\text {th }}$ position of any amino acid sequence can be defined as

$$
S_{n}=\left[\prod_{i=1}^{6} \delta_{n+4-i}\right] *(0.37)^{-6}
$$


Where $\delta_{\mathrm{n}}$ stands for fractional surface probability of an amino acid located at position $\mathrm{n}$. The probability value greater than 1.0 indicates the tendency of a residue to be located at the surface of protein. The hydrophilicity profile of the amino-acid sequence SARS-CoVid2 s-protein was predicted by correlating the hydrophilicity HPLC (High-performance liquid chromatography) parameters of twenty synthetic peptides ${ }^{5}$. The antigenicity of a peptide is believed to be depending on the flexibility of the side chain of the amino-acid residues ${ }^{10}$. The B-factors (temperature factor) of the residues present in S-protein is known to be necessary for measuring the segmental flexibility. The normalized B-value of $\mathrm{C}_{\alpha}$ atom of residue was calculated by the following equation

$$
B_{\text {norm }}=\left(B+D_{P}\right) /\left(<B>_{P}+D_{P}\right)
$$

Where, $<\mathrm{B}_{\mathrm{P}}>$ is the average $\mathrm{B}$-value of all $\mathrm{C}_{\alpha}$ atoms of protein $\mathrm{P}$ and $\mathrm{D}_{\mathrm{P}}$ is the tuning factor of the particular protein. The values of $\mathrm{B}_{\text {norm }}$ more than 1.0 indicates the antigenic region of a protein. Further, the antigenic propensity of the S-protein residues was calculated from the relation mentioned below

$$
A_{P}=f_{A g} / f_{S}
$$

Where, $f_{A g}$ stands for the frequency of occurrence of each amino-acids at the known antigenic regions and $f_{s}$ indicates the frequency of occurrence of the residues at the surface of query protein ${ }^{7}$. Further, the continuous residue stretches with higher $\beta$-turn, $\beta$-sheet propensities were also determined to identify the most antigenic regions of S-protein. VaxiJen $2.0^{11}$ was employed to evaluate the antigenicity of the predicted epitopes. The antigenic score of the predicted epitopes with an antigenicity score of 0.4 believed to initiate adequate defensive immune reaction. B-cell discontinuous or conformational epitope that are scattered along the protein sequence were identified by DiscoTope $2.0^{9}$ with a discotope score threshold of -3.7 was used to predict discontinuous epitopes. The Pymol was used to examine the positions of selected linear and discontinuous epitopes on the 3D structure of SARS-CoV-2 spike-protein.

A total of 30 peptides were predicted by Emini Surface accessibility method (Table S1) which may act as continuous B-cell epitopes. The residue wise surface accessibility profile is displayed in Table S2. Among them E144-153, E674-685, E773-779, E1139-1162 peptides are found to have high surface accessibility profile, hence considered as probable B-cell epitope (Figure S3-A). Next, we determined the hydrophilic profile of the residues of S-protein employing the method proposed by Parker et. al and depicted in Table S3. The continuous peptides E250-256, E254-257, E601-607, E745-751, E773-779, E774-780, E1256-1262, E1257-1263 found to have higher hydrophilicity score which indicates their possibility to interact with neutralizing antibody. The side chain flexibility of amino-acid residues of a protein are known to be crucial parameter to determine the antigenic region. The peptides obtained from Karplus \& Schulz flexibility method is given in Table S4. The peptides E71-77, E72-78, E110-116, E179-185, E180-186, E250-256, E251-257, 
E252-258, E412-418, E552-558, E600-606, E601-607, E675-681, E676-682, E677-683, E808-814, E809-815, E810-816 show high flexibility which have greater probability to interact with immune molecules. Further, 68 peptides were predicted by BepiPred method as linear B-cell epitope (Table S5). E249-261, E675-687, E805-816, E1256-1265 peptides showed high antigenic scores among the peptides predicted by BepiPred method. Similarly, 46 peptides are predicted by Kolaskar \& Tongaonkar method (Table S7) and the antigenicity profile of S-protein residues is given in Table S8. The linear epitopes E4-18, E125-134, E505-527, E1057-1070, E1221-1256 showed higher antigenicity score. Lastly, the $\beta$-sheet propensity profile of S-protein is calculated by Chou-Fasman method and displayed in Table S9. The continuous peptides E250-256, E251-257, E252-258, E253259, E476-482, E600-606, E807-813, E808-814, E1245-1251, E1246-1252, E1247-1254 showed higher $\beta$-sheet propensity, thus considered as linear B-cell epitopes. Furthermore, we used VaxiJen tool for assessing the antigenicity of the above-mentioned epitopes to filter the highly antigenic linear peptides and depicted in Table S10.

The conformational epitopes of the spike protein of COVID-19 in their closed and open state is determined by Disctope ( $\mathrm{V}-1.1)$ server and the threshold is set to -7.7. The regions above the threshold line was considered as conformational epitope.

\section{T-cell epitope prediction}

The development of memory B-cell in human body depends on the recognition of cytotoxic Tlymphocyte (CTL) epitopes presented at the surface of T-cell receptors. The memory B-cell is believed to fight against the re-infection (secondary immune response) to mitigate the effects of disease-causing pathogen ${ }^{12}$. IEDB tool was employed to predict the peptides that are presented by the major types of T-cell receptors (TCRs) such as Major Histocompatibility complex Class I (MHC Class I) and Class II. The prediction of both HLA (human leukocyte antigen) class-I and class-II Tcell epitopes are mainly based on artificial neural network (ANN) implemented in NetMHCpan tool $^{13,14}$. In the present case we considered $A * 02: 01, A * 24: 01, B * 40: 01, B * 58: 01$ alleles for MHC-I based and DRB1*04:01, DRB1*07:01 for MHC-II based epitopes due to their importance in case of SARS-CoVid2 ${ }^{15}$. In order to filter the T-cell epitope peptides with lower affinity, the Percentile rank threshold was set to $1 \%$ for MHC-I and 10\% for MHC-II. Further, the antigenicity of each T-cell epitopes was calculated by VaxiJen ${ }^{11}$ (v2.0).

We retrieved only the sequences which have lowest percentile rank of 0.1 . In case of A*02:01 allele, total 21 peptides such as E6-16, E116-126, E119-127, E192-203, E222-231, E224233, E444-452, E495-503, E509-517, E567-576, E584-592, E610-623, E626-635, E886-894, E972980, E976-985, E1007-1015, E1048-1061, E1059-1068, E1144-1152, E1181-1189 found to have lower percentile rank i.e higher affinity towards MHC-I based TCRs (Table S-13). Similarly, the continuous residue stretches E55-65, E76-86, E143-153, E160-168, E164-177, E170-177, E230-238, 
E317-329, E327-335, E368-378, E395-407, E422-429, E444-456, E504-517, E816-828, E885-894, E887-896, E899-906 show greater affinity towards HLA-A*24:02 (Table S-14). Further, the MHC-I molecule express from the HLA-B*40:01 and HLA-B*58:01 showed affinity towards E327-335, E505-513, E772-780, E893-902, 1143-1152 (Table-S15) and E19-28, E93-101, E195-204, E343-351, E636-643, E685-697, E781-789, E878-888, E890-898, E1086-E1096, E1091-1102 (Table-S16) epitope peptide respectively. The HLA alleles that are associated with MHC-II based SARS-CoVid2 peptide presentation are reported to be DRB1*04:01 and DRB1*07:01. The peptides with lowest percentile rank have been considered here due to higher affinity towards MHC-II molecules (Table S$17 \&$ S-18).

\section{Multi-epitope Vaccine design}

The continuous B-cell epitopes from S-protein predicted by different physicochemical methods mentioned above were linked together by GPGPG linkers in sequential manner. In order to induce regulatory immune response, the Cholera Toxin B (CTB) adjuvant was fused by EAAAK linker at the $\mathrm{N}$-terminal end of the vaccine construct. The similar procedure was followed for MHC-I and MHC-II based linear T-cell epitopes. The tertiary structure of the two linear vaccine construct was generated by trRosetta ${ }^{16}$ web server (https://yanglab.nankai.edu.cn/trRosetta/). Furthermore, the 3D-models of the vaccine was validated by ProSA-webserver ${ }^{17}$ (https://prosa.services.came.sbg.ac.at/prosa.php) and Ramachandran plot implemented in Schrodinger tool.

\section{Protein-protein Docking}

The interaction of epitopes with their respective receptor was evaluated by epitope-protein docking. All the docking calculations were carried out with open source $\mathrm{Hex}^{18}$ software. The humanized monoclonal antibody ${ }^{19}$ (PDB ID: 7BZ5, $1.84 \AA$ ) isolated from COVID-19 patient was used as a receptor for the B-cell epitopes. A grid-box was created at the light chain $\left(\mathrm{V}_{\mathrm{L}}\right)$ and heavy chain $\left(\mathrm{V}_{\mathrm{H}}\right)$ of the variable region of the neutralizing antibody. Care was taken to ensure the suitable accommodation of the designed vaccine and the conformational B-cell epitopes at the surface of the $\mathrm{V}_{\mathrm{L}}$ and $\mathrm{V}_{\mathrm{H}}$. Similarly, in case of T-cell epitopes we considered the 3D-structure of HLA-class I T-cell receptors (TCRs) such as A*02:01 (PDB ID: 2GTZ, $1.7 \AA$ ), A*24:01 (PDB ID: 5WWJ, $2.29 \AA$ ), B*40:01 (PDB ID: 6IEX, $2.31 \AA$ ), $\mathrm{B} * 58: 01$ (PDB ID: 5IM7, $2.5 \AA$ ) and HLA-class II T-cell receptor

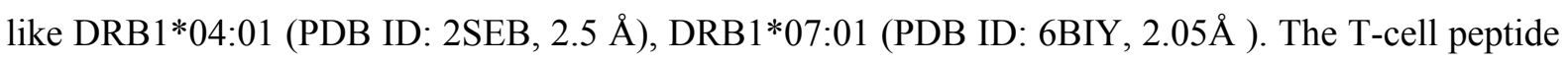
epitopes were constructed in Maestro workspace and docked at the peptide presenting pockets of the TCRs. Prior to docking the multiepitope linear vaccine, the conformational B-cell epitopes, T-cell peptide epitopes and the respective receptors were prepared using Protein-preparation wizard of Schrodinger tool. The protonation state of all the proteins was generated at the $\mathrm{pH}$ of 7.0 and the protein structures were minimized until the RMSD of the heavy atom converges up to $0.3 \AA$. The 
interactions of the vaccine with the receptors were visualized and analysed in Pymol software. Further the epitope-protein complexes were subjected to MD simulation for assessing their dynamic stability.

\section{Protocol}

We carried out atomistic MD simulations of all the systems using GROMACS-2016.520-22. Amber 99$\mathrm{SB}^{23}$ force-field was employed due to its better balance in $\beta$-sheet and helicity. propensity compared to other force fields ${ }^{24-26}$. SPC/E ${ }^{27}$ water molecules were used to solvate the receptor-peptide systems due to its compatibility with AMBER force field. All the systems underwent a 50000 step energy minimization by steepest-descent algorithm ${ }^{28,29}$ to remove the steric clash. Leapfrog integrator algorithm was used to integrate the equation of motions with a time step of $2 \mathrm{fs}$. LINCS ${ }^{30}$ algorithm was applied to constrain all the bonds in the peptide molecule and SETTLE algorithm was employed to constrain the geometry of water molecules. Next, the systems were equilibrated in canonical ensemble (NVT) followed by isothermal-isobaric ensemble (NPT) for 5 and 10 ns respectively by restraining the solute heavy atoms. Next, the restrain were removed and the protein molecules were allowed to move freely during the production run for $100 \mathrm{~ns}$. The temperature and pressure of the system was maintained employing Velocity $\operatorname{rescale}^{31}\left(\tau_{\mathrm{t}}=0.1 \mathrm{ps}\right)$ and Parrinello-Rahman coupling algorithm ${ }^{32}\left(\tau_{\mathrm{p}}=0.2 \mathrm{ps}\right)$. The cut-off for short range electrostatic and van der Waals interactions was assigned to $1.2 \mathrm{~nm}$. Particle Mesh Ewald method ${ }^{33,34}$ was used to calculate the long range electrostatic interactions. In order to mimic the environment of the outer cell surface $s$ we have done all the simulations at $\mathrm{pH}$ 7.0. In all the simulations, the coordinates were saved at every $10 \mathrm{ps}$ interval for analysis.

\section{MM/PBSA Free Energy Calculation}

The quantitative measure of the binding strength of docked epitopes to the allosteric pocket of immune receptors was computed by $\mathrm{MM} / \mathrm{PBSA}^{35}$ method. The average binding free energy of the epitopes can be defined as

$$
\Delta G_{\text {bind }}=\Delta G_{\text {Complex }}-\left(\Delta G_{\text {Protein }}+\Delta G_{\text {Epitope }}\right)
$$

$\Delta G_{\text {Complex }}$ is the Gibbs free energy of bound protein, whereas $\Delta G_{\text {Protein }}$ and $\Delta G_{\text {Epitope }}$ are individual Gibbs free energy of the unbound protein and epitope molecule. In general, $\Delta G_{\text {bind }}$ to form a proteinprotein complex can be written as ${ }^{36}$

$$
\begin{gathered}
\Delta G_{b i n d}=\left\langle\Delta G_{b i n d-M M}\right\rangle+\left\langle\Delta G_{b i n d-s o l}\right\rangle-T\langle\Delta S\rangle \\
\Delta G_{b i n d-M M}=\Delta G_{b i n d-e l e}+\Delta G_{b i n d-v d w}+\Delta G_{b i n d-c o v} \\
\Delta G_{b i n d-s o l}=\Delta G_{b i n d-P B}+\Delta G_{b i n d-S A}
\end{gathered}
$$

where, $\Delta G_{M M}$ is the total molecular mechanics energy of the interactions between protein and epitope. $\Delta G_{M M}$ is obtained from internal energy (bond, angle, dihedral energies), electrostatic $\left(\Delta G_{\text {bind-ele }}\right)$ and van der Waals energy $\left(\Delta G_{\text {bind-vdw }}\right)$ terms. The solvation free energy $\left(\Delta G_{\text {bind-sol }}\right)$ mainly includes 
electrostatic solvation energy and non-polar solvation energy terms. The electrostatic solvation free energy was calculated using Poisson Boltzmann (PB) method $^{37-39}$ and the non-polar contribution to solvation free energy $\left(\Delta G_{\text {bind-SA }}\right)$ was computed using solvent accessible surface area ${ }^{40,41}$. It is believed that implicit solvent models implicitly include entropy associated with the solvent during the calculation of solvation free energies. However, entropic contribution can also be calculated using normal mode analysis ${ }^{42}$. The last $10 \mathrm{~ns}$ of each simulation is considered for MM/PBSA calculation. The dielectric constant of solute and solvent is set to 2,80 .

\section{In-silico Cloning of Vaccine Construct}

The reverse translation along with the codon optimization of the vaccine constructs were carried out with Codon Adaptation tool ${ }^{43}$ (http://www.jcat.de/) in order to generate the cDNA sequence of the vaccines. The K-12 strain of E.coli was used to express the vaccines. The expression level of the vaccines was evaluated by codon adaptation index (CAI) and overall GC content. Finally, the cDNA sequence of the vaccines was inserted into pUC19 (2686 bp) employing SnapGene tool (http://www.snapgene.com).

The size of the vaccine construct consists of b-cell linear epitope (Vac-COVID-B) is found to be 399 base pair long whereas; the vaccine construct made of T-cell linear peptide epitopes (VacCOVID-T) is 333 base pair (bp) (SFigure ). The CAL value $>0.8$ and GC composition between 30 $70 \%$ of a cDNA segment is believed to be the benchmark for good expression of the gene in the host system ${ }^{46}$. The CAI value of both vaccines are found to be 1.0 and the GC content values for VacCOVID-B, Vac-COVID-T are $60.65 \%, 56.75 \%$ respectively. This indicates the efficient expression of the adapted codons in E.coli K12 strain. Finally, we inserted the cDNA sequences of the vaccines computationally at the multiple cloning site of pUC19 vector. The restriction map of the designed plasmid is shown in figure $\mathrm{S} 7$. The size of the recombinant plasmid is $3401 \mathrm{bp}$ and can be named as pUC-COVID. This study implies an efficient cloning strategy for the chimeric vaccines.

\section{Reference}

(1) Kumar, S.; Stecher, G.; Tamura, K. MEGA7: Molecular Evolutionary Genetics Analysis Version 7.0 for Bigger Datasets. Molecular Biology and Evolution 2016, 33 (7), 1870-1874.

(2) Kumar, S.; Tamura, K.; Jakobsen, I. B.; Nei, M. MEGA2: Molecular Evolutionary Genetics Analysis Software. Bioinformatics 2001, 17 (12), 1244-1245.

(3) Larkin, M. A.; Blackshields, G.; Brown, N. P.; Chenna, R.; McGettigan, P. A.; McWilliam, H.; Valentin, F.; Wallace, I. M.; Wilm, A.; Lopez, R.; Thompson, J. D.; Gibson, T. J.; Higgins, D. G. Clustal W and Clustal X Version 2.0. Bioinformatics 2007, 23 (21), 2947-2948.

(4) Emini, E.; Hughes, J.; Perlow, D.; Boger, J. Induction of Hepatitis A Virus-Neutralizing Antibody by a Virus-Specific Synthetic Peptide. Journal of virology 1985.

(5) Parker, J. M. R.; Guo, D.; Hodges, R. S. New Hydrophilicity Scale Derived from HighPerformance Liquid Chromatography Peptide Retention Data: Correlation of Predicted Surface 
Residues with Antigenicity and x-Ray-Derived Accessible Sites. Biochemistry 1986, 25 (19), $5425-5432$.

(6) Karplus, P. A.; Schulz, G. E. Prediction of Chain Flexibility in Proteins. Naturwissenschaften 1985, 72 (4), 212-213.

(7) Kolaskar, A. S.; Tongaonkar, P. C. A Semi-Empirical Method for Prediction of Antigenic Determinants on Protein Antigens. FEBS Lett. 1990, 276 (1-2), 172-174.

(8) Chou, P. Y.; Fasman, G. D. Secondary Structural Prediction of Proteins from Their Amino Acid Sequence. Trends in Biochemical Sciences 1977, 2 (6), 128-131.

(9) Larsen, J. E. P.; Lund, O.; Nielsen, M. Improved Method for Predicting Linear B-Cell Epitopes. Immunome Res 2006, 2, 2.

(10) Westhof, E.; Altschuh, D.; Moras, D.; Bloomer, A. C.; Mondragon, A.; Klug, A.; Van Regenmortel, M. H. V. Correlation between Segmental Mobility and the Location of Antigenic Determinants in Proteins. Nature 1984, 311 (5982), 123-126.

(11) Doytchinova, I. A.; Flower, D. R. VaxiJen: A Server for Prediction of Protective Antigens, Tumour Antigens and Subunit Vaccines. BMC Bioinformatics 2007, 8, 4.

(12) Akkaya, M.; Kwak, K.; Pierce, S. K. B Cell Memory: Building Two Walls of Protection against Pathogens. Nature Reviews Immunology 2020, 20 (4), 229-238.

(13) Nielsen, M.; Andreatta, M. NetMHCpan-3.0; Improved Prediction of Binding to MHC Class I Molecules Integrating Information from Multiple Receptor and Peptide Length Datasets. Genome Medicine 2016, 8 (1), 33.

(14) Andreatta, M.; Nielsen, M. Gapped Sequence Alignment Using Artificial Neural Networks: Application to the MHC Class I System. Bioinformatics 2016, 32 (4), 511-517.

(15) Grifoni, A.; Sidney, J.; Zhang, Y.; Scheuermann, R. H.; Peters, B.; Sette, A. A Sequence Homology and Bioinformatic Approach Can Predict Candidate Targets for Immune Responses to SARS-CoV-2. Cell Host \& Microbe 2020, 27 (4), 671-680.e2.

(16) Yang, J.; Anishchenko, I.; Park, H.; Peng, Z.; Ovchinnikov, S.; Baker, D. Improved Protein Structure Prediction Using Predicted Interresidue Orientations. PNAS 2020, 117 (3), 14961503.

(17) Wiederstein, M.; Sippl, M. J. ProSA-Web: Interactive Web Service for the Recognition of Errors in Three-Dimensional Structures of Proteins. Nucleic Acids Res 2007, 35 (suppl_2), W407-W410.

(18) Macindoe, G.; Mavridis, L.; Venkatraman, V.; Devignes, M.-D.; Ritchie, D. W. HexServer: An FFT-Based Protein Docking Server Powered by Graphics Processors. Nucleic Acids Res 2010, 38 (suppl_2), W445-W449.

(19) Wu, Y.; Wang, F.; Shen, C.; Peng, W.; Li, D.; Zhao, C.; Li, Z.; Li, S.; Bi, Y.; Yang, Y. et al. A Noncompeting Pair of Human Neutralizing Antibodies Block COVID-19 Virus Binding to Its Receptor ACE2. Science 2020, 368 (6496), 1274-1278.

(20) Spoel, D. V. D.; Lindahl, E.; Hess, B.; Groenhof, G.; Mark, A. E.; Berendsen, H. J. C. GROMACS: Fast, Flexible, and Free. Journal of Computational Chemistry 2005, 26 (16), $1701-1718$.

(21) Abraham, M. J.; Murtola, T.; Schulz, R.; Páll, S.; Smith, J. C.; Hess, B.; Lindahl, E. GROMACS: High Performance Molecular Simulations through Multi-Level Parallelism from Laptops to Supercomputers. SoftwareX 2015, 1-2, 19-25.

(22) Berendsen, H. J. C.; van der Spoel, D.; van Drunen, R. GROMACS: A Message-Passing Parallel Molecular Dynamics Implementation. Computer Physics Communications 1995, 91 (1), 43-56.

(23) Schmid, N.; Eichenberger, A. P.; Choutko, A.; Riniker, S.; Winger, M.; Mark, A. E.; van Gunsteren, W. F. Definition and Testing of the GROMOS Force-Field Versions 54A7 and 54B7. Eur Biophys $J$ 2011, 40 (7), 843.

(24) Man, V. H.; He, X.; Derreumaux, P.; Ji, B.; Xie, X.-Q.; Nguyen, P. H.; Wang, J. Effects of AllAtom Molecular Mechanics Force Fields on Amyloid Peptide Assembly: The Case of A $\beta 16-$ 22 Dimer. J. Chem. Theory Comput. 2019, 15 (2), 1440-1452.

(25) Chamachi, N. G.; Chakrabarty, S. Replica Exchange Molecular Dynamics Study of Dimerization in Prion Protein: Multiple Modes of Interaction and Stabilization. J. Phys. Chem. B 2016, 120 (30), 7332-7345. 
(26) Best, R. B.; Buchete, N.-V.; Hummer, G. Are Current Molecular Dynamics Force Fields Too Helical? Biophysical Journal 2008, 95 (1), L07-L09.

(27) Mark, P.; Nilsson, L. Structure and Dynamics of the TIP3P, SPC, and SPC/E Water Models at 298 K. J. Phys. Chem A 2001, 105 (43), 9954-9960.

(28) Averill, F. W.; Painter, G. S. Steepest-Descent Determination of Occupation Numbers and Energy Minimization in the Local-Density Approximation. Phys. Rev. B 1992, 46 (4), 24982502.

(29) Meza, J. C. Steepest Descent. WIREs Computational Statistics 2010, 2 (6), 719-722.

(30) Hess, B.; Bekker, H.; Berendsen, H. J. C.; Fraaije, J. G. E. M. LINCS: A Linear Constraint Solver for Molecular Simulations. Journal of Computational Chemistry 1997, 18 (12), 14631472.

(31) Bussi, G.; Donadio, D.; Parrinello, M. Canonical Sampling through Velocity Rescaling. J. Chem. Phys. 2007, 126 (1), 014101.

(32) Parrinello, M.; Rahman, A. Polymorphic Transitions in Single Crystals: A New Molecular Dynamics Method. Journal of Applied Physics 1981, 52 (12), 7182-7190.

(33) Essmann, U.; Perera, L.; Berkowitz, M. L.; Darden, T.; Lee, H.; Pedersen, L. G. A Smooth Particle Mesh Ewald Method. J. Chem. Phys. 1995, 103 (19), 8577-8593.

(34) Darden, T.; York, D.; Pedersen, L. Particle Mesh Ewald: An N·log(N) Method for Ewald Sums in Large Systems. J. Chem. Phys. 1993, 98 (12), 10089-10092.

(35) Kumari, R.; Kumar, R.; Lynn, A. G_mmpbsa-A GROMACS Tool for High-Throughput MM-PBSA Calculations. J. Chem. Inf. Model. 2014, 54 (7), 1951-1962.

(36) Kollman, P. A.; Massova, I.; Reyes, C.; Kuhn, B.; Huo, S.; Chong, L.; Lee, M.; Lee, T.; Duan, Y.; Wang, W. et al. Calculating Structures and Free Energies of Complex Molecules: Combining Molecular Mechanics and Continuum Models. Accounts of Chemical Research 2000, 33 (12), 889-897.

(37) Honig, B.; Nicholls, A. Classical Electrostatics in Biology and Chemistry. Science 1995, 268 (5214), 1144-1149.

(38) Srinivasan, J.; Cheatham, T. E.; Cieplak, P.; Kollman, P. A.; Case, D. A. Continuum Solvent Studies of the Stability of DNA, RNA, and Phosphoramidate-DNA Helices. J. Am. Chem. Soc. 1998, 120 (37), 9401-9409.

(39) Baker, N. A.; Sept, D.; Joseph, S.; Holst, M. J.; McCammon, J. A. Electrostatics of Nanosystems: Application to Microtubules and the Ribosome. PNAS 2001, 98 (18), 1003710041.

(40) Hermann, R. B. Theory of Hydrophobic Bonding. II. Correlation of Hydrocarbon Solubility in Water with Solvent Cavity Surface Area. The Journal of Physical Chemistry 1972, 76 (19), 2754-2759.

(41) Amidon, G. L.; Yalkowsky, S. H.; Anik, S. T.; Valvani, S. C. Solubility of Nonelectrolytes in Polar Solvents. V. Estimation of the Solubility of Aliphatic Monofunctional Compounds in Water Using a Molecular Surface Area Approach. The Journal of Physical Chemistry 1975, 79 (21), 2239-2246.

(42) Wang, J.; Hou, T.; Xu, X. Recent Advances in Free Energy Calculations with a Combination of Molecular Mechanics and Continuum Models. Current Computer Aided-Drug Design 2006, $2(3), 287-306$.

(43) Grote, A.; Hiller, K.; Scheer, M.; Münch, R.; Nörtemann, B.; Hempel, D. C.; Jahn, D. JCat: A Novel Tool to Adapt Codon Usage of a Target Gene to Its Potential Expression Host. Nucleic Acids Res 2005, 33 (suppl_2), W526-W531. 